General Mathematics Vol. 27, No. 1 (2019), 37-43

DOI:10.2478/gm-2019-0004

S sciendo

\title{
Notes on certain p-valent functions ${ }^{1}$
}

\author{
Mugur Acu, Shigeyoshi Owa
}

\begin{abstract}
Let $A_{p}$ be the class of functions $f(z)$ which are analytic in the open unit disk $U$. Applying some subordinations for functions, some interesting properties for $f(z) \in A_{p}$ are showed. Our results are generalizations for results given before.
\end{abstract}

2010 Mathematics Subject Classification: 30C45, 30C80.

Key words and phrases: analytic functions, $p$-valent functions, univalent functions, subordination.

\section{Introduction}

Let $A_{p}$ be the class of functions $f(z)$ of the form:

$$
f(z)=z^{p}+\sum_{k=1}^{\infty} a_{p+k} z^{p+k}(p \in \mathbb{N}=\{1,2,3, \ldots\})
$$

which are analytic in the open unit disk $U=\{z: z \in \mathbb{C}$ and $|z|<1\}$.

For functions $f(z)$ and $g(z)$ which are analytic in $U$, we say that $f(z)$ is subordinate to $g(z)$ if there exists a function $w(z)$ which is analytic in $U$ with $w(0)=0$ and $|w(z)|<1, z \in U$, and such that $f(z)=g(w(z))$. We write this subordination by

$$
f(z) \prec g(z), z \in U \text {. }
$$

If $g(z)$ is analytic and univalent in $U$, then the subordination (2) is equivalent to $f(0)=g(0)$ and $f(U) \subset g(U)$. For the subordinations, Miller and Mocanu [2] gave the following lemma.

\footnotetext{
${ }^{1}$ Received 4 April, 2019

Accepted for publication (in revised form) 8 May, 2019
} 
Lemma 1.1 If a function $p(z)$ is analytic in $U$ with $p(0)=1$ and satisfies the following subordination

$$
z p^{\prime}(z) \prec z, z \in U,
$$

then $p(z)$ satisfies

$$
p(z) \prec 1+z, z \in U .
$$

The function $1+z$ is the best dominant for (3).

Applying the above lemma, Ozaki and Nunokawa [5] have shown

Theorem 1.1 If a function $f(z) \in A_{1}$ satisfies

$$
\operatorname{Re}\left(\frac{f^{2}(z)}{z^{2} f^{\prime}(z)}\right) \geq \frac{1}{2}, z \in U,
$$

then $f(z)$ is univalent in $U$.

Remark 1.1 It is easy to see that the inequality (5) is equivalent to

$$
\left|\frac{z^{2} f^{\prime}(z)}{f^{2}(z)}-1\right| \leq 1, z \in U
$$

Furthermore, Nunokawa, Obradović and Owa [4] have given the following result.

Theorem 1.2 If a function $f(z) \in A_{1}$ satisfies $\frac{f(z)}{z} \neq 0,0<|z|<1$ and

$$
\left|\left(\frac{z}{f(z)}\right)^{\prime \prime}\right| \leq 1, z \in U
$$

then $f(z)$ is univalent in $U$.

In this present paper, we try to consider some generalizations for Theorem 1.1 and Theorem 1.2.

\section{Applications of subordinations}

Our first application of subordinations for $f(z) \in A_{p}$ is the following.

Theorem 2.1 If a function $f(z) \in A_{p}$ satisfies

$$
\left|\left(\frac{z^{p-j}}{f^{(j)}(z)}\right)^{\prime \prime}\right| \leq \frac{(p-j) !(p-j)(1-\alpha)}{p !}, z \in U
$$


for some real $\alpha(0 \leq \alpha<1)$ and $j=0,1,2, \ldots, p-1$, then

$$
\left|\frac{p ! z^{p+1-j} f^{(j+1)}(z)}{(p-j) !(p-j)\left(f^{(j)}(z)\right)^{2}}-1\right|<1-\alpha, z \in U,
$$

and that

$$
\operatorname{Re}\left(\frac{\left(f^{(j)}(z)\right)^{2}}{z^{p+1-j} f^{(j+1)}(z)}\right)>\frac{p !}{(p-j) !(p-j)(2-\alpha)}, z \in U .
$$

Proof. For $f(z) \in A_{p}$ satisfying (8), we define a function $p(z)$ by

$$
\begin{gathered}
p(z)=\frac{\frac{p ! z^{p+1-j} f^{(j+1)}(z)}{(p-j) !(p-j)\left(f^{(j)}(z)\right)^{2}}-\alpha}{1-\alpha} \\
=\frac{1}{1-\alpha}\left\{\frac{p ! z^{p-j}}{(p-j) ! f^{(j)}(z)}-z\left(\frac{p ! z^{p-j}}{(p-j) !(p-j) f^{(j)}(z)}\right)^{\prime}-\alpha\right\} .
\end{gathered}
$$

Then $p(z)$ is analytic in $U$ with $p(0)=1$ and

$$
z p^{\prime}(z)=\frac{-z^{2}}{1-\alpha}\left(\frac{p ! z^{p-j}}{(p-j) !(p-j) f^{(j)}(z)}\right)^{\prime \prime} .
$$

With our condition (8), we see that

$$
\begin{gathered}
\left|z p^{\prime}(z)\right|=\left|\frac{-z^{2}}{1-\alpha}\left(\frac{p ! z^{p-j}}{(p-j) !(p-j) f^{(j)}(z)}\right)^{\prime \prime}\right| \\
\leq|z|^{2}<1, z \in U .
\end{gathered}
$$

This give us that

$$
z p^{\prime}(z)=\frac{-z^{2}}{1-\alpha}\left(\frac{p ! z^{p-j}}{(p-j) !(p-j) f^{(j)}(z)}\right)^{\prime \prime} \prec z, z \in U .
$$

Therefore, applying Lemma 1.1 for $p(z)$, we obtain that

$$
p(z)=\frac{\frac{p ! z^{p+1-j} f^{(j+1)}(z)}{(p-j) !(p-j)\left(f^{(j)}(z)\right)^{2}}-\alpha}{1-\alpha} \prec 1+z, z \in U .
$$

It follows from (15) that

$$
\frac{p ! z^{p+j-1} f^{(j+1)}(z)}{(p-j) !(p-j)\left(f^{(j)}(z)\right)^{2}}-\alpha \prec(1-\alpha)(1+z), z \in U,
$$


that is, that

$$
\frac{p ! z^{p+j-1} f^{(j+1)}(z)}{(p-j) !(p-j)\left(f^{(j)}(z)\right)^{2}}-1 \prec(1-\alpha) z, z \in U .
$$

This subordination (17) gives us the inequality (9). Further, it is easy to see that the inequality (9) is equivalent to (10).

Making $j=0$ in Theorem 2.1, we have

Corollary 2.1 If $f(z) \in A_{p}$ satisfies

$$
\left|\left(\frac{z^{p}}{f(z)}\right)^{\prime \prime}\right| \leq p(1-\alpha), z \in U
$$

for some real $\alpha, 0 \leq \alpha<1$, then

$$
\left|\frac{z^{p+1} f^{\prime}(z)}{p f^{2}(z)}-1\right|<1-\alpha, z \in U,
$$

and that

$$
\operatorname{Re}\left(\frac{f^{2}(z)}{z^{p+1} f^{\prime}(z)}\right)>\frac{1}{p(2-\alpha)}, z \in U .
$$

Further, letting $p=1$ in Corollary 2.1, we see

Corollary 2.2 If $f(z) \in A_{1}$ satisfies

$$
\left|\left(\frac{z}{f(z)}\right)^{\prime \prime}\right| \leq 1-\alpha, z \in U
$$

for some real $\alpha, 0 \leq \alpha<1$, then

$$
\left|\frac{z^{2} f^{\prime}(z)}{f^{2}(z)}-1\right|<1-\alpha, z \in U,
$$

and $f(z)$ is univalent in $U$.

Remark 2.1 If we consider $\alpha=0$ in Corollary 2.2, then we have Theorem 1.2 by Nunokawa, Obradović and Owa [4].

Open Problem With Corollary 2.1, we have the following open problem. For $f(z) \in A_{p}$ satisfying (18), can we say that $f(z)$ is univalent in $U$ ?

By Corollary 2.2, if $p=1$ in Corollary 2.1, we have $f(z)$ is univalent in $U$.

Next, we introduce Lemma 2 due to Hallenbeck and Ruscheweyh [1] (or Miller and Mocanu [3]). 
Lemma 2.1 Let a function $h(z)$ be convex in $U$ with $h(0)=1$ and $p(z)$ be analytic in $U$ with $p(0)=1$. If $p(z)$ satisfies the subordination

$$
p(z)+z p^{\prime}(z) \prec h(z), z \in U,
$$

then

$$
p(z) \prec q(z) \prec h(z), z \in U,
$$

where

$$
q(z)=\frac{1}{z} \int_{0}^{z} h(t) d t
$$

The function $q(z)$ is convex in $U$ and the best dominant of (24).

Applying the above lemma, we derive

Theorem 2.2 If a function $f(z) \in A_{p}$ satisfies

$$
\frac{p !}{(p-j) !(p-j)}\left\{\frac{z^{p+1-j} f^{(j+1)}(z)}{\left(f^{(j)}(z)\right)^{2}}-z^{2}\left(\frac{z^{p-j}}{f^{(j)}(z)}\right)^{\prime \prime}\right\} \prec \frac{1+(1-2 \alpha) z}{1-z}, z \in U
$$

for some real $\alpha(0 \leq \alpha<1)$, then

$$
\frac{z^{p+1-j} f^{(j+1)}(z)}{\left(f^{(j)}(z)\right)^{2}} \prec q(z), z \in U
$$

where

$$
q(z)=-\frac{(p-j) !(p-j)}{p !}\left\{\frac{2(1-\alpha)}{z} \log (1-z)+1-2 \alpha\right\}
$$

and $q(z)$ is the best dominant.

Proof. Defining the function $p(z)$ by (11), we have $z p^{\prime}(z)$ which is given by (12). Considering a function $h(z)$ given by

$$
h(z)=\frac{1+z}{1-z}, z \in U,
$$

we know that $h(z)$ is analytic and convex in $U$.

We apply Lemma 2.1 for such $p(z)$ and $h(z)$. Then Lemma 2.1 gives us that

$$
p(z)+z p^{\prime}(z) \prec h(z), z \in U
$$

implies the subordination

$$
p(z) \prec q(z) \prec h(z), z \in U
$$


with $q(z)$ given by (25).

Wee see that the subordination (30) is equivalent to (26). Further, it follows that

$$
\frac{1}{z} \int_{0}^{z} \frac{1+t}{1-t} d t=\frac{1}{z} \int_{0}^{z}\left(\frac{2}{1-t}-1\right) d t=\frac{2}{z} \log \frac{1}{1-z}-1
$$

Therefore, the subordination(31) becomes

$$
p(z) \prec \frac{2}{z} \log \left(\frac{1}{1-z}\right)-1 \prec \frac{1+z}{1-z}, z \in U .
$$

From (33), we have that

$$
\frac{p ! z^{p+1-j} f^{(j+1)}(z)}{(p-j) !(p-j)\left(f^{(j)}(z)\right)^{2}} \prec \alpha+(1-\alpha)\left(\frac{2}{z} \log \left(\frac{1}{1-z}\right)-1\right), z \in U,
$$

that is, that

(35)

$$
\frac{z^{p+1-j} f^{(j+1)}(z)}{\left(f^{(j)}(z)\right)^{2}} \prec-\frac{(p-j) !(p-j)}{p !}\left\{\frac{2(1-\alpha)}{z} \log \left(\frac{1}{1-z}\right)+1-2 \alpha\right\}, z \in U .
$$

This completes the proof of the theorem.

Letting $j=0$ in Theorem 2.2, we have

Corollary 2.3 If $f(z) \in A_{p}$ satisfies

$$
\frac{z^{p+1} f^{\prime}(z)}{f^{2}(z)}-z^{2}\left(\frac{z^{p}}{f(z)}\right)^{\prime \prime} \prec p \frac{1+(1-2 \alpha) z}{1-z}, z \in U
$$

for some real $\alpha(0 \leq \alpha<1)$, then

$$
\frac{z^{p+1} f^{\prime}(z)}{f^{2}(z)} \prec p\left(\frac{2(1-\alpha)}{z} \log \left(\frac{1}{1-z}\right)+2 \alpha-1\right), z \in U .
$$

Making $\alpha=\frac{1}{2}$ in Corollary 2.3, we obtain

Corollary 2.4 If $f(z) \in A_{p}$ satisfies

$$
\frac{z^{p+1} f^{\prime}(z)}{f^{2}(z)}-z^{2}\left(\frac{z^{p}}{f(z)}\right)^{\prime \prime} \prec \frac{p}{1-z}, z \in U,
$$

then

$$
\frac{z^{p+1} f^{\prime}(z)}{f^{2}(z)} \prec \frac{p}{z} \log \left(\frac{1}{1-z}\right), z \in U
$$


Finally, putting $p=1$ in Corollary 2.3, we see

Corollary 2.5 If $f(z) \in A_{1}$ satisfies

$$
\frac{z^{2} f^{\prime}(z)}{f^{2}(z)}-z^{2}\left(\frac{z}{f(z)}\right)^{\prime \prime} \prec \frac{1+(1-2 \alpha) z}{1-z}, z \in U
$$

for some real $\alpha(0 \leq \alpha<1)$, then

$$
\frac{z^{2} f^{\prime}(z)}{f^{2}(z)} \prec \frac{2(1-\alpha)}{z} \log \left(\frac{1}{1-z}\right)+2 \alpha-1, z \in U \text {. }
$$

\section{References}

[1] D. J. Hallenbeck, St. Ruscheweyh, Subordination by convex functions, Proc. Amer. Math. Soc., vol. 52, 1975, 191-195.

[2] S. S. Miller, P. T. Mocanu, On some classes of first-order differential subordinations, Michigan Math. J., vol. 32, 1985, 185-195.

[3] S. S. Miller, P. T. Mocanu, Differential Subordinations, Theory and Applications, Marcel Dekker, 2000.

[4] M. Nunokawa, M. Obradović, S. Owa, One criterion for univalency, Proc. Amer. Math. Soc., vol. 106, 1989, 1035-1037.

[5] S. Ozaki, M. Nunokawa, The Schwarzian derivative and univalent functions, Proc. Amer. Math. Soc., vol. 33, 1972, 392-394.

\section{Mugur Acu}

Lucian Blaga University of Sibiu

Faculty of Science

Department of Mathematics and Informatics

Street Dr. I. Raţiu 5-7, 550012 Sibiu, Romania

e-mail: acu_mugur@yahoo.com mugur.acu@ulbsibiu.ro

\section{Honorary Professor Shigeyoshi Owa}

1 Decembrie 1918 University of Alba Iulia

Alba Iulia, Romania

e-mail: shige21@ican.zaq.ne.jp 